\title{
Screening for Suitable Prebiotic for Probiotic Strain by in vitro Fermentation
}

\author{
Kalpa E Rengarajan and Preetha Radhakrishnan* \\ Department of Food Process Engineering,SRM University, Kattankulathur - 603203, India. \\ http://dx.doi.org/10.13005/bbra/2149
}

(Received: 17 March 2016; accepted: 06 May 2016)

\begin{abstract}
Prebiotics are components of food that promote the growth of colon bacteria. As they constitute of non-digestible fibre compounds, they pass unaffected through the upper part of the gastro-intestinal tract and selectively promote the growth of colon bacteria. In this project food such as corn, oats, oat bran and asparagus were screened for their prebiotic ability by testing their effect on the growth of potential probiotic Lactobacillus species. This was carried out by using in vitro fermentation where MRS medium was modified to constitute the prebiotic food. It was found that growth of the Lactobacillus strain was promoted significantly more by corn $(13.62 \log \mathrm{CFU} / \mathrm{mL})$ than the other three prebiotic samples. Correspondingly, the rate of acidification of the medium containing corn, by the Lactobacillus was significantly higher ( $\mathrm{pH} 4.12$ ) than the media containing the other three prebiotic samples.
\end{abstract}

Keywords: probiotic, prebiotics, Lactobacillus, in vitro fermentation.

Probiotics are live microorganisms which when consumed in specific amounts provide health benefits to the consumer ${ }^{1}$. Probiotics mostly consist of Lactobacilli and Bifidobacteria. Lactobacilli are presently being consumed in the form of functional foods and their valuable effects have been established in several studies ${ }^{2}$. It is a fundamental requirement that these bacterial should be able to resist the harsh gastro-intestinal conditions where they are subjected to amylases in the oral cavity, acidity in the stomach, bile fluids and pancreatic juice in the duodenum; and there have been several in vivo and in vitro studies that have shown that Lactobacilli have not been able to withstand such harsh conditions and have only partially survived ${ }^{3}$. It is also crucial that probiotic

\footnotetext{
* To whom all correspondence should be addressed. E-mail : preetha.r@ktr.srmunic.ac.in
}

products have a minimum of $10^{6} \mathrm{CFU} / \mathrm{mL}$ of the bacteria at the time of expiry due to the minimum dosage per day being $10^{8}-10^{9} \mathrm{CFU} / \mathrm{mL}^{4}$. Many Lactobacilli strains are susceptible to the environment of the gastro-intestinal tract. There have been several studies where the addition of certain compounds have improved the resistance of the bacteria to the human disgestive environment. Chen, Chen, Liu, Lin \& Chiu (2005) ${ }^{5}$ reported that the addition of the prebiotics fructooligosaccharides and isomaltooligoaccharides as coating materials while encapsulating the bacteria improved its survival rate and provided protection. Picot and Lacroix $(2004)^{6}$ reported that the utilization of whey protein for immobilisation improved the cell survival when subjected to extreme conditions. Valerio et al.(2006) ${ }^{7}$ reported the use of artichokes as a prebiotic for several probiotic strains which acted as the carbohydrate source and also protected the bacteria in the gastrointestinal tract. 
A prebiotic is "a selectively fermented ingredient that allows specific changes, both in the composition and/or activity in the gastrointestinal microbiota that confers benefits upon host wellbeing and health"”. Colon bacteria, mostly Bifidobacteria and Lactobacilli, are chosen are targetsto enhance the gut health due to their health-benefitting effects. This is done by the application of prebiotics which are indigestible fibre compounds and can only be fermented by the colon bacteria in the body ${ }^{9}$. Current research has established the various health benefits of prebiotics such as maintenance of gut health, cancer prevention, immunity boosting, cholesterol reduction, obesity and constipation prevention, reduction of risk of cardiovascular diseases, bacteriocin production and many more ${ }^{10}$. Macfarlane, Macfarlane \& Cummings, (2006) ${ }^{11}$ reported that the prebiotics such as oligo-fructose, galacto-ologosaccharides and lactulose significantly changed the bowel microflora by improving the growth of Bifidobacteria and Lactobacilli.

Prebiotics mostly include oligosaccharides which are found in a variety of plant sources. It is essential to investigate potential prebiotic oligosaccharides from sources that are easily available. These sources include oats, oat bran, corn and asparagus. Oats and oat bran are a rich source of â-D-glucan ${ }^{12}$, corn is rich in xylooligosaccharides ${ }^{13}$ and asparagus in rich in inulin and fructooligoaccharides ${ }^{14}$.

The beneficial effect of prebiotics along with probiotics has brought about symbiotics which refers to products that contain both prebiotics and probiotics where the prebiotic

Table 1. Acidification of L.rhamnosus in minimal media containing the prebiotics as carbon sources

\begin{tabular}{lll}
\hline Sl no. & Prebiotics & $\mathrm{pH}$ \\
\hline 1 & Oats & $4.52^{\mathrm{a}} \pm 0.02$ \\
2 & Oat bran & $5.33^{\mathrm{b}} \pm 0.025$ \\
3 & Corn & $4.12^{\mathrm{c}} \pm 0.025$ \\
4 & Asparagus & $5.63^{\mathrm{d}} \pm 0.03$ \\
5 & Minimal medium & $6.5^{\mathrm{e}} \pm 0.02$ \\
\hline
\end{tabular}

Values are representatives of mean \pm S.D. $(n=$ $3)$. Values followed by different superscripts in a column are significantly different $(\mathrm{P}<0.05)$. components are selectively utilized by the probiotic bacteria ${ }^{15}$. Knowledge about this selective utilisation is important as individual strains of bacteria are very preferential towards their substrate $^{16,17}$. Bifidobacterium strains are preferential towards xylose based oligo- and polysaccharides, and are able to utilize them resourcefully to promote their own growth ${ }^{18}$. Hence, it is crucial that while formulating a symbiotic product the right prebiotic is chosen for the probiotic.

In the present study, oats, oat bran, corn and asparagus were screened for their prebiotic ability against potential probiotic Lactobacillus strain. This was carried by in vitro fermentation where the food samples were incorporated into carbon free MRS medium. The growth rate and the acidification rate were determined for the Lactobacillus strain when it was subjected to each of the food samples. This helped in identifying the complementary prebiotic for the strain which can further be utilised to formulate a symbiotic product.

\section{MATERIALSANDMETHODS}

\section{Sources of microorganisms and prebiotics}

Lactobacillus strain with potential probiotic propertieswas obtained from MTCC, Chandigarh in the lyophilised form. de Man, Ragosa and Sharpe (MRS) medium was used to revive and maintain the bacterial culture.

The bacterial cells were cultivated in MRS for 24 hours at 37p C. Cells were then harvested by centrifugation at $2500 \times \mathrm{g}$ at $4 \mathrm{p} \mathrm{C}$ and were washed and resuspended in saline (0.9\%). This inoculums was utilised for further studies. Fresh inoculums was always prepared and utilised.

Oats, oat bran, corn and asparagus were selected to test their ability as potential prebiotic for the selected probiotic strain. Oats and oat bran were sorted to remove dust particles. Asparagus and corn were washed thoroughly with water and dried.

\section{Incorporation of prebiotic into the media}

Media was prepared according to the method suggested by Hernandez-Hernandez et al. $(2012)^{19}$ with slight modifications. Prebiotic was incorporated into carbohydrate free, MRS basal medium which consisted of preoteose peptone $(10 \mathrm{~g} / \mathrm{L})$, beef extract $(5 \mathrm{~g} / \mathrm{L})$, yeast extract $(10 \mathrm{~g} / \mathrm{L})$, 
ammonium citrate $(2 g / L)$, sodium acetate $(5 g / L)$, magnesium sulphate $(0.1 \mathrm{~g} / \mathrm{L})$, manganese sulphate $(0.05 \mathrm{~g} / \mathrm{L})$, dipotassium phosphate $(2 \mathrm{~g} / \mathrm{L})$ and polysorbate $80(1 \mathrm{~g} / \mathrm{L})$. The prebiotic samples were cooked with minimal amounts of water and filtered with a muslin cloth. The filtrate was obtained and $2 \mathrm{~g} / \mathrm{L}$ of the filtrate was added to the media in place of dextrose to provide a carbon source.

\section{Selection of complementary prebiotic}

Selection of complementary prebiotic was carried out by in vitro fermentation where the growth of theLactobacillus strain, when inoculated into the media containing each of the prebiotic samples, was observed. The media with the prebiotic samples were inoculated with the bacteria and incubated at 37p C for 48 hours, after which the viable count was determined by plating onto MRS agar. The rate of acidification was also carried by using a $\mathrm{pH}$ meter ${ }^{20}$. All experiments were carried out in triplicates.

\section{Statistical analysis}

The data were subjected to one-way analysis of variance (ANOVA) and the significance of the difference between means was determined by Duncan's multiple range test $(\mathrm{P}<0.05)$. Values are expressed as means of triplicate determinants \pm standard deviation.

\section{RESULTS AND DISCUSION}

Growth of the Lactobacillus strain was variable when subjected to the different prebiotic samples. There was a significant difference among the growth influenced by the prebiotics - oats, oat bran, corn and asparagus (Fig 1). Very limited growth was observed in the medium sans the carbon source which acted as the control. This proved that the four prebiotics used in this research are good sources of carbon. Corn was found to promote the growth of the Lactobacillus strain significantly better than oats, oat bran and asparagus at $13.62 \log \mathrm{CFU} / \mathrm{mL}$. The acidification rate of the prebiotics - shown in table 1 - due to fermentation by the probiotic strain, also corresponded with the growth. The $\mathrm{pH}$ in the medium containing corn at $\mathrm{pH} 4.12$ was significantly lower than the other three media while the $\mathrm{pH}$ of the control media remained almost the same.

Prebiotics consist of various oligosaccharides in varying concentrations. Oats and oat bran consist of â-D-glucan, corn consists of xylooligosaccharides and asparagus consists of inulin-type fructans. Voragen (1998) ${ }^{21}$ reported that the variation in the composition of prebioticmight be the reason for the difference in the growth rates. The fermentation rates of oligosaccharides are influenced by sugar and glycosidic linkages and branching, cooperation among bacteria during fermentation, nature of fermentation, relationship among the bacteria, its substrates and fermentation products, and the degree of polymerisation. Xylooligosaccharides have a degree of polymerisation in the range of 210 and have the capacity to multiply the number of probiotics and even suppress the growth of Clostridium $^{22}$. Jaskari et al. $(1998)^{23}$ reported that
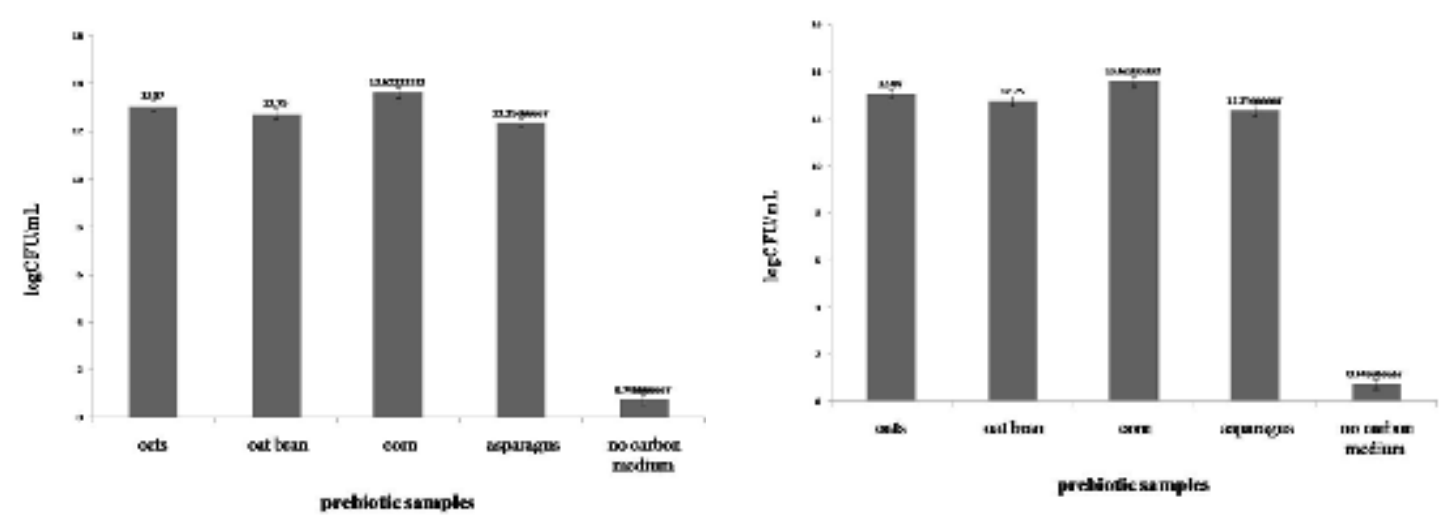

Growth was measured in terms of $\log \mathrm{CFU} / \mathrm{mL}$. Error bars indicate standard deviation of means $(\mathrm{n}=3)$

Fig. 1. Selection of complementary prebiotic by fermentation 
Lactobacilli are preferential towards oligosaccharides other than xylooligosaccharides as substrates but in this study it was found that corn promoted the growth of the Lactobacillus the best. Zampa et al.(2004) ${ }^{24}$ reported that the beneficial effects of xylooligosaccharides also arise from their ability to reduce the concentrations of secondary bile acids which increase the risk of cancer in the colon.

\section{CONCLUSION}

In the present study, oat, oat bran, corn and asparagus were tested for their prebiotic ability against potential prebiotic strain Lactobacillus rhamnosus. This was done to determine and select a complementary prebiotic for the probiotic. Future studies are to be carried out where their symbiotic nature can be further exploited. Co-encapsulation may be utilised to entrap the probiotic and prebiotic together to bring about synergism, and can be added to foods to fortify them.

\section{ACKNOWLEDGEMENT}

The authors would like to thank the Dean of Engineering and the Dean of Bioengineering, of SRM University for permitting us to pursue this project. We would also like to thank the Department of Food Process Engineering for helping us in successfully completing this project.

\section{REFERENCES}

1. Probiotics in food: health and nutritional properties and guidelines for evaluation. Food and Agriculture Organization of the United Nations, 2006.

2. Reid G. Probiotics and prebiotics-progress and challenges. International Dairy Journal. 2008, 30;18(10):969-75.

3. Hernandez-Hernandez O, Muthaiyan A, Moreno FJ, Montilla A, Sanz ML, Ricke SC. Effect of prebiotic carbohydrates on the growth and tolerance of Lactobacillus. Food Microbiology. 2012; 30(2):355-61.

4. Kailasapathy K, Chin J. Survival and therapeutic potential of probiotic organisms with reference to Lactobacillus acidophilus and Bifidobacterium spp. Immunology and Cell Biology. 2000; 78(1):80-8.

5. Chen KN, Chen MJ, Liu JR, Lin CW, Chiu HY.
Optimization of incorporated prebiotics as coating materials for probiotic microencapsulation. Journal of food science. 2005; 70(5):M260-6.

6. Picot A, Lacroix C. Encapsulation of bifidobacteria in whey protein-based microcapsules and survival in simulated gastrointestinal conditions and in yoghurt. International Dairy Journal. 2004; 14(6):50515.

7. Valerio F, De Bellis P, Lonigro SL, Morelli L, Visconti A, Lavermicocca P. In vitro and in vivo survival and transit tolerance of potentially probiotic strains carried by artichokes in the gastrointestinal tract. Applied and environmental microbiology. 2006; 72(4):3042-5.

8. Gibson GR, Probert HM, Van Loo J, Rastall RA, Roberfroid MB. Dietary modulation of the human colonic microbiota: updating the concept of prebiotics. Nutrition research reviews. 2004; 17(02):259-75.

9. Charalampopoulos D, Rastall RA. Prebiotics in foods. Current opinion in biotechnology. 2012; 23(2):187-91.

10. Patel S, Goyal A. The current trends and future perspectives of prebiotics research: a review. 3 Biotech. 2012 ; 2(2):115-25.

11. Macfarlane SM, Macfarlane GT, Cummings JT. Review article: prebiotics in the gastrointestinal tract. Alimentary pharmacology \& therapeutics. 2006; 24(5):701-14.

12. Kontula P, von Wright A, Mattila-Sandholm T. Oat bran â-gluco-and xylo-oligosaccharides as fermentative substrates for lactic acid bacteria. International journal of food microbiology. 1998; 45(2):163-9.

13. Moura P, Barata R, Carvalheiro F, Gírio F, Loureiro-Dias MC, Esteves MP. In vitro fermentation of xylo-oligosaccharides from corn cobs autohydrolysis by Bifidobacterium and Lactobacillus strains. LWT-Food Science and Technology. 2007; 40(6):963-72.

14. Shiomi N, Yamada J, Izawa M. Isolation and identification of fructo-oligosaccharides in roots of asparagus (Asparagus officinalis L.). Agricultural and Biological Chemistry. 1976; 40(3):567-75.

15. Schrezenmeir J, de Vrese M. Probiotics, prebiotics, and synbiotics-approaching a definition. The American journal of clinical nutrition. 2001; 73(2):361s-4s.

16. Crittenden R, Karppinen S, Ojanen S, Tenkanen M, Fagerström R, Mättö J, Saarela M, Mattila Sandholm T, Poutanen K. In vitro fermentation of cereal dietary fibre carbohydrates by probiotic and intestinal bacteria. Journal of the Science of 
Food and Agriculture. 2002; 82(8):781-9.

17. Hopkins MJ, Englyst HN, Macfarlane S, Furrie E, Macfarlane GT, McBain AJ. Degradation of cross-linked and non-cross-linked arabinoxylans by the intestinal microbiota in children. Applied and environmental microbiology. 2003; 69(11):6354-60.

18. Iyer C, Kailasapathy K. Effect of co encapsulation of probiotics with prebiotics on increasing the viability of encapsulated bacteria under in vitro acidic and bile salt conditions and in yogurt. Journal of Food Science. 2005 ; 70(1):M18-23.

19. Voragen AG. Technological aspects of functional food-related carbohydrates. Trends in Food Science \& Technology. 1998; 9(8):328-35.
20. Izumi K, Azumi N. Xylooligosaccharide compositions useful as food and feed additives. Japan Patent JP. 2001; 2(001):226-409.

21. Jaskari J, Kontula P, Siitonen A, Jousimies-Somer H, Mattila-Sandholm T, Poutanen K. Oat âglucan and xylan hydrolysates as selective substrates for Bifidobacterium and Lactobacillus strains. Applied Microbiology and Biotechnology. 1998; 49(2):175-81.

22. Zampa A, Silvi S, Fabiani R, Morozzi G, Orpianesi C, Cresci A. Effects of different digestible carbohydrates on bile acid metabolism and SCFA production by human gut micro-flora grown in an in vitro semi-continuous culture. Anaerobe. 2004; 10(1):19-26. 